\title{
Index autorum ad Vol. 150
}

Confecit F. Ammann, Geneve

(B) = Buchbesprechungen - Livres nouveaux - Book reviews. (R) = Referate von Vorträgen, die an anderer Stelle ausführlich erschienen sind oder erscheinen werden. Die Autorennamen wurden in diesem Index autorum nicht aufgenommen. - Rapports de conferences qui ont été (ou seront) publiées ailleurs in extenso. Cet Index autorum ne contient pas les noms des auteurs. Abstract

$\mathrm{s}$ of lectures that have been (or will be) published in extenso elsewhere. The names of the authors have not been included in

this Index autorum.

Auricchio, G., 196, 452

Barraquer, M. J. I., Ill

Batra, D. V., 107

Becker, P. E. (Editor), 92 (B)

Bellmann, O., 138

Benck, P., 46

Benkö, S., 270

Bettelheim, H., 241 Bietti, G. B., 18 Bocci, N., 196, 452 Branemark, P. I., 239 (B) Burau, J., 259

Caldeira, J. A. F., 285 Cameron, M. E., 240 (B)

468 Index a

Casanovas, J., 239 (B) Cernea, P., 409 Cuendet, J. F., 92 (B)

Daniele, S., 196, 452 Dekking, H. M., 153 Deller, M., 76 De Rouck, A., 83

Ebner, R., 422 Eisner, G., 371

Fialho, S. A., 318 (B) Fiore, C, 196, 452 Floares, G., 409 Francois, J., 83, 215

Gailloud, CL, 92 (B), 157 (B) Gerhard, J.-P., 46 Gemet, H., 386 Girard, L. J., 161 Goldmann, H., 65 Grieten, J., 36 Gutman, F., 5

Harrington, D. O., 158 (B) Hockwin, O., 187 Holland, G., 138 Hollwich, 318 (B) Hughes, W. F., 318 (B)

Imamura, S., 240 (B) Irimiciuc, E., 409

Jäggy, W., 159 (B) Jones, D. H., 401 Jütte, A., 353

Kahán, A., 270 Kahán, I. L., 270 Klöti, R., 158 (B) Kutschera, E., 422

Laníni, P., 263 Lemke, L., 353 Licht, W., 187 Lobstein, A., 46

Martenet, A.-C, 97 McEwen, W. K., 321 Meier-Ruge, W., 127 Müller, 318 (B)

urn ad Vol. 150

Nécsei, P., 292 Neubauer, H., 441 Noll, E., 187 Nordmann, J., 46 Norton, E. W. D., 5 Notter, H., 252

Oktürk, I., 123 Ostachowicz, M., 259

Pannarale, M. R., 18 Papamatheakis, N., 347 Pau, H., 167 Pék, L., 292 Puckovskaja, W. A., 239

(B)

Radnot, M., 92 (B) 
Rák, K., 270

Ramos, A. O., 285

Ramos, L., 285

Rivara, A., 431

Rouck, A. de, v. De Rouck, A.

Sadkiewicz, A., 259

Schillaci, C., 306

Schmidt, Th., 65, 203

Scuderi, G., 306

Soper, J. W., 161

Stenström, S., 157 (B)

St. Helen, R,, 321

Straub, W., 57

Streiff, E. B., 1, 76, 157-159 (B),

239-240 (B),318 (B) Szmigielski, M., 83

Teng Khoen Hing, 219

Tost, M., 359

Trevor-Roper, P. D., 457-460 (R)

Velhagen, K., 158 (B|, 318 (B) Verrey, F., 97 Verriest, G., 83

W. Weale

R. A.

157 (B) Weekers

R.

36 Witmer

$\mathrm{R}$.

97 Wolter

J. R.

401 Wright

158 (B)

Zbranca

E.

409 Zingirian

M.

431 Zlatar

P.

175 